\title{
Histology and Glutamine Synthetase Immunoreactivity in Liver Biopsies From Patients With Congestive Heart Failure
}

\author{
Bela Horvath ${ }^{\mathrm{a}, \mathrm{g}}$, Lei Zhu ${ }^{\mathrm{b}, \mathrm{g}}$, Daniela Allende ${ }^{\mathrm{a}}$, Hao Xie ${ }^{\mathrm{c}}$, John Guirguis ${ }^{\mathrm{d}}$, Michael Cruise ${ }^{\mathrm{a}}$, \\ Deepa T. Patila ${ }^{\mathrm{a}}$, Robert O'Shead ${ }^{\mathrm{d}}$, John Rivas ${ }^{\mathrm{d}}$, Reyna Yordanka ${ }^{\mathrm{e}}$, \\ Nan Lan ${ }^{\mathrm{d}}$, Xiuli Liu ${ }^{\mathrm{f}, \mathrm{h}}$
}

\begin{abstract}
Background: Long-standing congestive heart failure can induce a constellation of histopathology changes in the liver that can range from mild sinusoidal dilation to advanced fibrosis and loss of normal perivenular expression of glutamine synthetase (GS). Liver biopsies might be performed to assess the perioperative risk of these patients or to determine the need of synchronous liver transplant. We aimed to assess interobserver agreement in recognizing these liver histologic features in patients undergoing evaluation for heart transplantation and to examine whether immunohistochemistry of GS will aid the diagnosis of cardiac hepatopathy $(\mathrm{CH})$.

Methods: Hematoxylin-eosin and trichrome-stained slides from 36 liver biopsies from patients undergoing evaluation for heart transplantation were reviewed by four liver pathologists. Histologic features of $\mathrm{CH}$ were reviewed and an overall fibrosis (stage) was assessed according to a recently proposed congestive hepatic fibrosis score (CHFS). In addition, 24 liver biopsies with a consensus diagnosis of $\mathrm{CH}$ and eight liver biopsies with no significant pathological changes were subjected to immunohistochemistry for GS. The Fleiss' kappa coefficient $(\mathrm{K})$ analysis was performed to determine the interobserver agreement. Further, histologic features of $\mathrm{CH}$ were correlated with the staining pattern of GS.
\end{abstract}

Results: Sinusoidal dilation, centrilobular hepatocyte atrophy, centrilobular fibrosis and hemorrhage were the most common findings in this cohort with a substantial-to-fair level of interobserver agree-

Manuscript submitted May 28, 2017, accepted June 6, 2017

aDepartment of Anatomic Pathology, Cleveland Clinic, Cleveland, OH, USA bDepartment of Gastroenterology, The first Affiliated Hospital, Dalian Medical University, Dalian City, China

${ }^{\mathrm{c}}$ Department of Medicine, Yale University, New Haven, CT, USA

dDepartment of Gastroenterology and Hepatology, Cleveland Clinic, Cleveland, $\mathrm{OH}, \mathrm{USA}$

eDepartment of Cardiology, Cleveland Clinic, Weston, FL, USA

fDepartment of Pathology, Immunology and Laboratory Medicine, University of Florida, Gainesville, FL, USA

gThese authors contributed equally to this article.

${ }^{\mathrm{h}}$ Corresponding Author: Xiuli Liu, Department of Anatomic Pathology, Immunology and Laboratory Medicine, University of Florida, PO Box 100275, Gainesville, FL 32610, USA. Email: xiuliliu@ufl.edu

doi: https://doi.org/10.14740/gr875e ment among four reviewers. The overall agreement on the diagnosis of $\mathrm{CH}$ and $\mathrm{CHFS}$ was moderate $(\mathrm{K}=0.55,95 \%$ confidence interval (CI): 0.32 - 0.73) and fair $(\mathrm{K}=0.35,95 \% \mathrm{CI}: 0.24-0.49)$, respectively. Twelve (of $24,50 \%$ ) cases of $\mathrm{CH}$ showed loss of the normal perivenular GS staining, while the remaining 12 cases of $\mathrm{CH}$ and all eight controls showed retained GS expression. Histologic features of $\mathrm{CH}$ (presence of sinusoidal dilation, centrilobular hepatocyte atrophy, hemorrhage, and centrilobular fibrosis) and the stage of fibrosis (CHFS) were not correlated with the loss of GS staining.

Conclusion: Most common features of $\mathrm{CH}$ can be interpreted with fair-to-substantial level of agreement by liver pathologists, with an overall moderate level agreement for the diagnosis and fair agreement for CHFS. Loss of normal perivenular expression of GS only occurs in $50 \% \mathrm{CH}$ and thus is not a sensitive marker for $\mathrm{CH}$.

Keywords: Cardiac hepatopathy; Glutamine synthetase; Liver biopsy; Congestive heart failure

\section{Introduction}

Liver pathology in patients with congestive heart failure is known as cardiac hepatopathy $(\mathrm{CH})$ or "nutmeg liver" which was initially described by Sherlock in 1951 in liver necropsy specimens [1]. It was characterized by sinusoidal dilatation and centrilobular hepatocyte necrosis. Later on, several studies further described these histologic findings in post-mortem liver specimens from patients with various cardiac diseases or shock, including centrilobular congestion, with or without necrosis, inflammation, sclerosis, and regenerative hyperplasia [2-4]. With the increasing use of heart transplant in the treatment of heart failure, liver biopsies are increasingly performed to assess the perioperative risk of these patients or to determine the need of synchronous liver transplant. Several studies suggested that chronic $\mathrm{CH}$ seems to be a benign, potentially reversible disease [5]. Myers et al reported that elevated right atrial pressure and hepatic venous pressure were associated with the presence of centrilobular necrosis, inflammation, and sinusoidal dilation, but not with hepatic fibrosis [6]. Thus, staging liver biopsies in this type of clinical setting is challenging, although progression to advanced-stage disease (cardiac cirrhosis) remains rare despite the common manifestation of 
Table 1. Features Examined in the Liver Biopsies in This Study

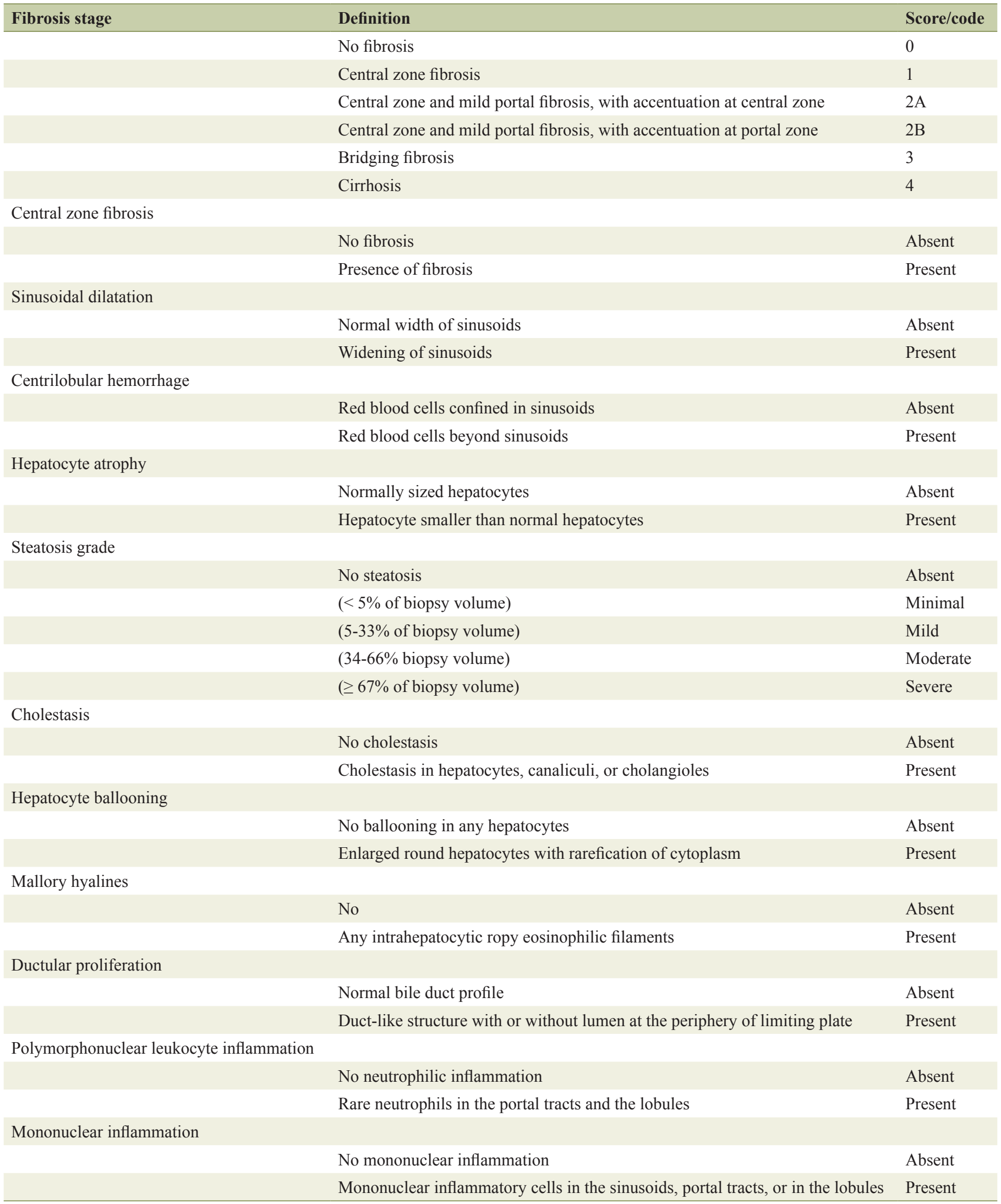


centrilobular fibrosis in patients with $\mathrm{CH}[2,6]$. Recently, one study proposed a congestive hepatic fibrosis score (CHFS) as a novel histologic assessment of clinical severity [7]. However, the interobserver agreement of this scoring system has not been tested in clinical setting.

Hepatic injury caused by severe heart failure usually results from prolonged recurrent congestion and/or impaired arterial perfusion. Hepatic injury is associated with biochemical alterations. For example, in normal human liver, glutamine synthetase (GS) is expressed in a rim of hepatocytes surrounding hepatic veins. GS expression is decreased in cirrhosis and efferent venous flow impairment and increased in chronic hepatitis, and focal nodular hyperplasia [8,9]. Decreased perivenular GS expression was reported in seven of seven liver specimens $(100 \%)$ from patients with congestive heart failure [9], suggesting that the loss of perivenular GS can be used as a marker for $\mathrm{CH}$. In addition, six (of seven) showed focal mild to moderate increase in GS positive hepatocyte progenitor cells (HPCs) [9].

We aimed to assess interobserver agreement in recognizing these liver histologic features in patients undergoing evaluation for heart transplantation and to examine whether immunohistochemistry of GS will aid the diagnosis of $\mathrm{CH}$.

\section{Methods}

Medical records at the Cleveland Clinic were searched for patients diagnosed with congestive heart failure from January 2000 to February 2015. Hematoxylin-eosin and trichromestained slides from 36 cases were retrieved and assessed by four liver pathologists. A consensus for each feature is defined as an interpretation agreed upon by three out of four pathologists. The adequacy of the biopsy specimen is defined as biopsy length of at least $1.5 \mathrm{~cm}$. All cases that were analyzed meet the criteria. Histologic features of congestive hepatopathy and other common liver histologic parameters were reviewed, including evaluation of the overall fibrosis (stage) using a recently developed CHFS [7] (Table 1). Briefly, based on the pattern of fibrosis, scores of $0,1,2 \mathrm{~A}, 2 \mathrm{~B}, 3$, and 4 were assigned as follows: score 0, no fibrosis; score 1, central fibrosis; score 2A, central zone and portal fibrosis, with accentuation of fibrosis in the central zone; score $2 \mathrm{~B}$, moderate portal fibrosis and central zone fibrosis, with accentuation of fibrosis in the portal zone; score 3, bridging fibrosis; score 4, cirrhosis.

Twenty-four liver biopsies were selected with a consensus diagnosis of $\mathrm{CH}$ and were stained with GS. Eight liver biopsies with no significant pathological changes from normal donor liver were included as controls. Immunohistochemistry for GS was performed on whole tissue section from formalin-fixed and paraffin-embedded tissue according to manufacturer's instructions using an automatic stainer (Benchmark, Ventana, Tucson, AZ, USA). Briefly, de-paraffinized tissue sections were stained with antibodies against GS (clone GS-6, at 1:500 working dilution; Millipore, Billerica, MA, USA) after antigen retrieval for 30 min using CC1 buffer (Ventana). We used diaminobenzidine as the chromogen. Appropriate positive and negative controls were reviewed. All immunohistochemical stains were evaluated by two pathologists (XL and BH) under a multiheaded microscope. Normal GS expression was defined as a rim of 2 - 3 layers of strongly positive hepatocytes around the hepatic veins (perivenular pattern). Absence of GS in the perivenular region was considered abnormal in this study. Immunoreactivity of GS in other regions was not assessed.

Fleiss' kappa was used to assess the interobserver agreement on each histologic feature and CHFS. A kappa value < $0,0.01-0.20,0.21-0.40,0.41-0.60,0.61-0.80$, and 0.81 - 1.0 is considered poor, slight, fair, moderate, substantial, and almost perfect agreement, respectively [10]. Kendall's taub correlation tests were performed to determine the correlation coefficients between histologic features of $\mathrm{CH}$ and the loss of perivenular GS immunoreactivity. A P-value $<0.05$ was considered statistically significant. Statistical analysis was performed using R 2.15.2 (R Development Core Team, 2012, Vienna, Austria).

\section{Results}

A total of 36 liver biopsies taken from patients with clinically and radiographically confirmed congestive heart failure were subjected to detailed histology review by four liver pathologists for the features listed in Table 1 and using criteria described previously [7]. When there was no consensus for a feature, it was categorized as indeterminate. In this cohort, $72 \%$ of cases had sinusoidal dilation, 69\% centrilobular fibrosis, 58\% hepatocyte atrophy, and $47 \%$ centrilobular hemorrhage (Figs. 1 and 2), consistent with previous studies that sinusoidal dilation, centrilobular hepatocyte atrophy, fibrosis and hemorrhage were the most common findings in $\mathrm{CH}$. Steatosis is only rare finding as $94 \%$ cases showed no steatosis or less than $5 \%$ of steatosis. Hepatocyte ballooning and cholestasis was noted in one case (3\%) each. Mononuclear inflammation was observed in the portal triads and lobules in 53\% and 36\% of biopsies. Ductular proliferation was noted in $36 \%$ of cases. Five cases had no fibrosis, while the remaining cases showed variable fibrosis including central zone fibrosis only $(n=4,11 \%)$, centrilobular and portal fibrosis with accentuation in central zone (n $=6,17 \%$ ), centrilobular and portal fibrosis with accentuation in portal zone $(n=5,14 \%)$, bridging fibrosis $(n=6,17 \%)$, cirrhosis $(\mathrm{n}=4,11 \%)$ and fibrosis of indeterminate degree $(\mathrm{n}=$ $6,17 \%)$. The consensus histologic data are shown in Table 2.

A fair-to-good level of interobserver agreement was reached among four reviewers on histopathological features (Table 3). Evaluation of sinusoidal dilatation had substantial interobserver agreement $(0.66,95 \% \mathrm{CI}: 0.41-0.86)$. The other features, centrilobular hepatocyte atrophy, centrilobular fibrosis, and centrilobular hemorrhage only had moderate to fair interobserver agreement. The overall agreement on the diagnosis of $\mathrm{CH}$ and $\mathrm{CHFS}$ was moderate $(\mathrm{K}=0.55,95 \% \mathrm{CI}: 0.32$ - 0.73$)$ and fair $(\mathrm{K}=0.35,95 \% \mathrm{CI}: 0.24-0.49)$, respectively. When the fibrosis stage is simplified into advanced ( 3 and 4 ) versus no or non-advanced fibrosis $(0-2 \mathrm{~B})$, the interobserver agreement improved to moderate $(\mathrm{K}=0.45,95 \% \mathrm{CI}$ : 0.31 $0.61)$.

Twenty-four liver biopsies had consensus diagnosis of $\mathrm{CH}$ 
Table 2. Consensus Histologic Characteristics of 36 Liver Biopsies From Patients With Cardiac Hepatopathy

\begin{tabular}{|c|c|}
\hline & $\mathbf{N}(\%)$ \\
\hline \multicolumn{2}{|l|}{ Stage of fibrosis } \\
\hline No fibrosis & $5(14)$ \\
\hline Centrilobular fibrosis only (1) & $4(11)$ \\
\hline Centrilobular (prominent) and portal fibrosis (2A) & $6(17)$ \\
\hline Centrilobular and portal (prominent) fibrosis (2B) & $5(14)$ \\
\hline Bridging fibrosis (3) & $6(17)$ \\
\hline Cirrhosis (4) & $4(11)$ \\
\hline Not cirrhosis, but with fibrosis of indeterminate degree & $6(17)$ \\
\hline \multicolumn{2}{|l|}{ Centrilobular fibrosis } \\
\hline Absent & $6(17)$ \\
\hline Present & $25(69)$ \\
\hline Indeterminate & $5(14)$ \\
\hline \multicolumn{2}{|l|}{ Sinusoidal dilatation } \\
\hline Absent & $8(22)$ \\
\hline Present & $26(72)$ \\
\hline Indeterminate & $2(6)$ \\
\hline \multicolumn{2}{|l|}{ Hepatocyte atrophy } \\
\hline Absent & $12(34)$ \\
\hline Present & $21(58)$ \\
\hline Indeterminate & $3(8)$ \\
\hline \multicolumn{2}{|l|}{ Centrilobular hemorrhage } \\
\hline Absent & $15(42)$ \\
\hline Present & $17(47)$ \\
\hline Indeterminate & $4(11)$ \\
\hline \multicolumn{2}{|l|}{ Steatosis } \\
\hline$<5 \%$ & $34(94)$ \\
\hline $5-33 \%$ & $1(3)$ \\
\hline $33-66 \%$ & $0(0)$ \\
\hline$>66 \%$ & $1(3)$ \\
\hline \multicolumn{2}{|l|}{ Ballooning } \\
\hline Absent & $35(97)$ \\
\hline Present & $1(3)$ \\
\hline \multicolumn{2}{|l|}{ Mallory hyalines } \\
\hline Absent & $36(100)$ \\
\hline Present & $0(0)$ \\
\hline \multicolumn{2}{|l|}{ Cholestasis } \\
\hline Absent & $35(97)$ \\
\hline Present & $1(3)$ \\
\hline \multicolumn{2}{|l|}{ Lobular polymorphonuclear leukocyte infiltration } \\
\hline Absent & $36(100)$ \\
\hline Present & $0(0)$ \\
\hline \multicolumn{2}{|l|}{ Lobular mononuclear inflammation } \\
\hline Absent & $21(58)$ \\
\hline Present & $13(36)$ \\
\hline Indeterminate & $2(6)$ \\
\hline \multicolumn{2}{|l|}{ Portal polymorphonuclear leukocyte infiltration } \\
\hline Absent & $35(97)$ \\
\hline Present & $1(3)$ \\
\hline \multicolumn{2}{|l|}{ Portal mononuclear inflammation } \\
\hline Absent & $10(28)$ \\
\hline Present & $19(53)$ \\
\hline Indeterminate & $7(19)$ \\
\hline \multicolumn{2}{|l|}{ Ductular proliferation } \\
\hline Absent & $7(20)$ \\
\hline Present & $13(36)$ \\
\hline Indeterminate & $16(44)$ \\
\hline
\end{tabular}




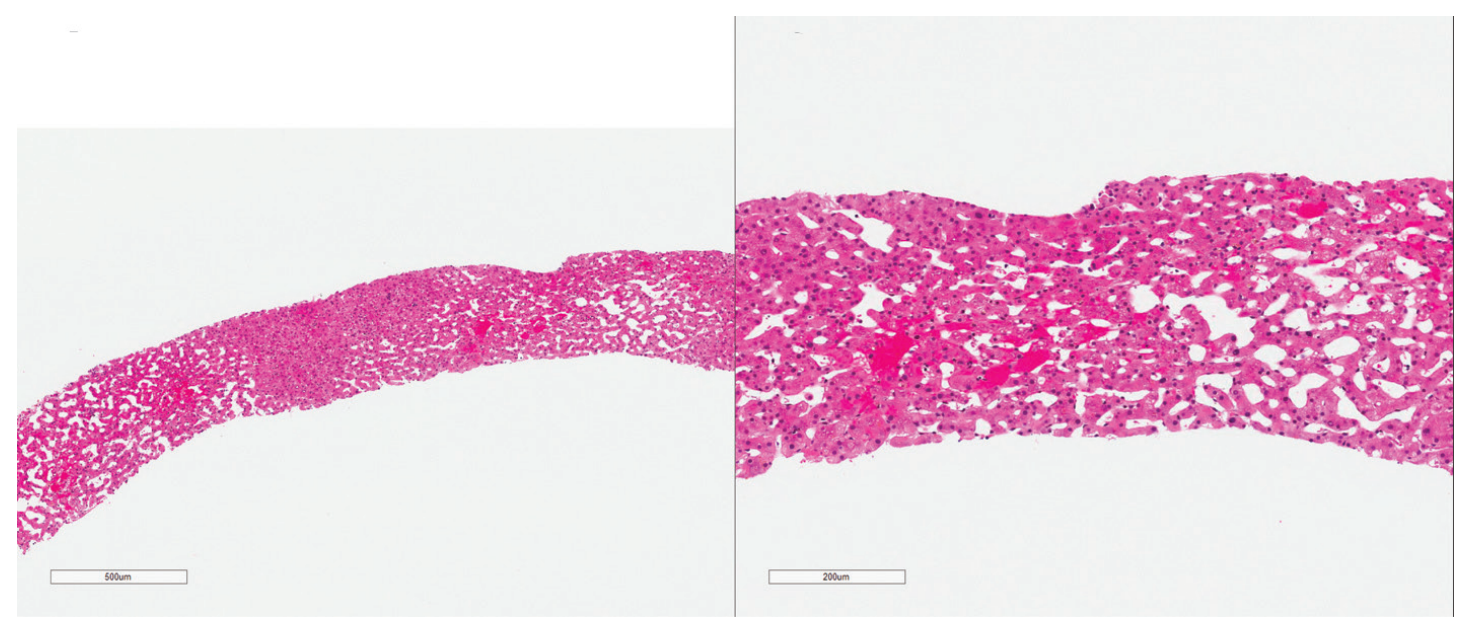

Figure 1. Morphologic features seen in congestive hepatopathy, including marked sinusoidal dilatation, centrilobular hepatocellular atrophy and hemorrhage (H\&E).

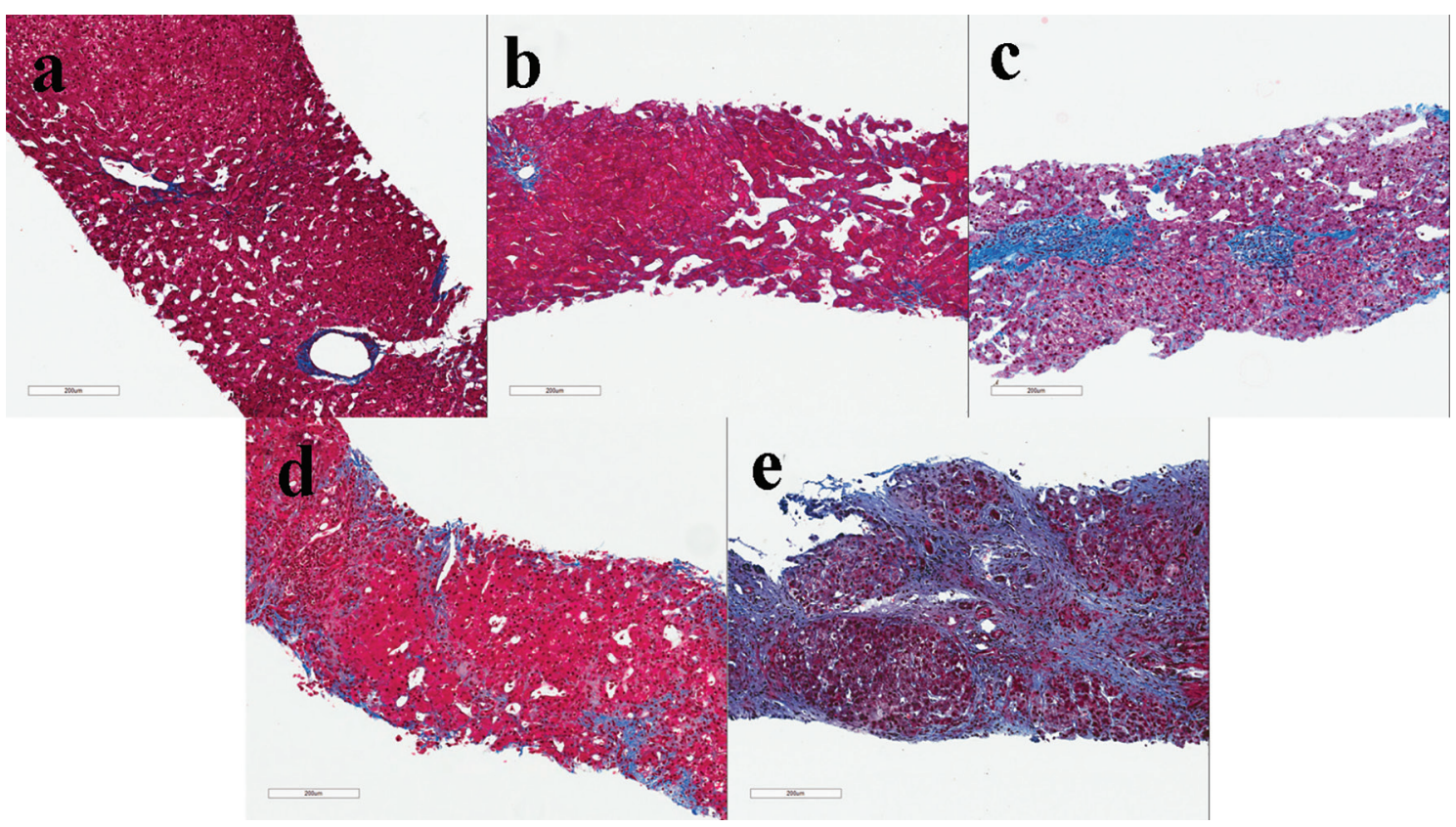

Figure 2. Examples of liver biopsies with a congestive hepatic fibrosis score 1, central fibrosis (a); score $2 \mathrm{~A}$, central zone and portal fibrosis, with accentuation of fibrosis in the central zone (b); score 2B, moderate portal fibrosis and central zone fibrosis, with accentuation of fibrosis in the portal zone (c); score 3, bridging fibrosis (d); score 4, cirrhosis (e) (Masson's trichrome stain).

Table 3. Interobserver Agreement on Histopathologic Features of Cardiac Hepatopathy

\begin{tabular}{lll}
\hline Histologic feature & K value (95\% CI) & Strength of agreement \\
\hline Sinusoidal dilation (absent/present) & $0.66(0.41-0.86)$ & Substantial \\
Centrilobular atrophy (absent/present) & $0.51(0.35-0.70)$ & Moderate \\
Centrilobular hemorrhage (absent/present) & $0.35(0.19-0.53)$ & Fair \\
Centrilobular fibrosis (absent/present) & $0.43(0.21-0.68)$ & Moderate \\
Fibrosis stage (congestive hepatic fibrosis score) & $0.35(0.24-0.49)$ & Fair \\
Simplified fibrosis stage (CHFS 0-2B/3-4) & $0.45(0.31-0.61)$ & Moderate \\
\hline
\end{tabular}




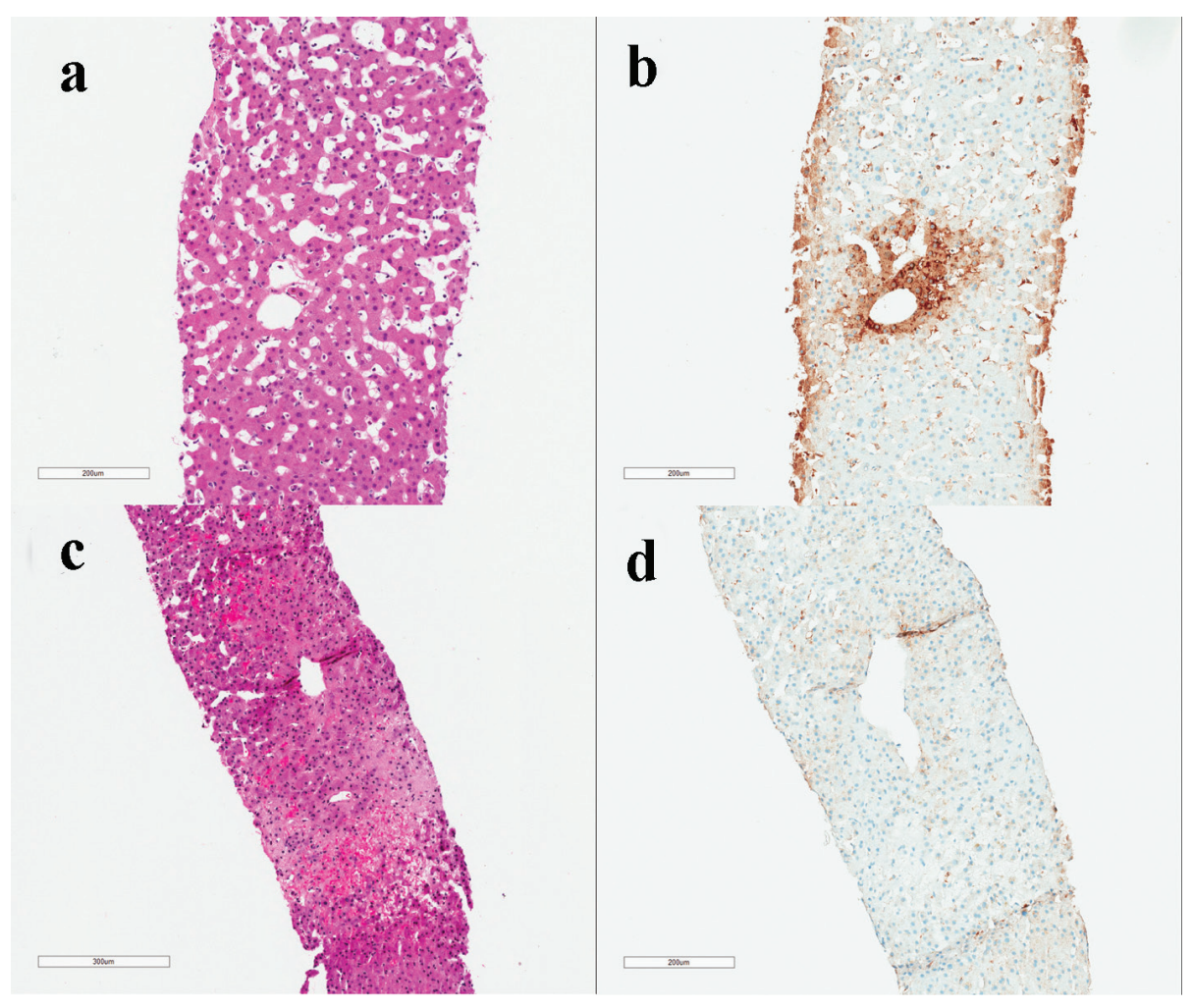

Figure 3. Examples of retained (b) and lost (d) normal perivascular pattern of glutamine synthetase staining (IHC with corresponding H\&E - a, c) in congestive hepatopathy.

and were subjected to immunohistochemistry for GS. All eight control cases showed strong perivenular GS staining. Twelve cases of $\mathrm{CH}$ showed retained and 12 cases of $\mathrm{CH}$ showed loss of the normal perivenular GS staining (Fig. 3). Histologic features of $\mathrm{CH}$ (presence of sinusoidal dilation, centrilobular hepatocyte atrophy, hemorrhage, and centrilobular fibrosis) and the stage of fibrosis (CHFS) did not correlate with the loss of normal GS staining pattern (Table 4).

\section{Discussion}

Congestive hepatopathy or $\mathrm{CH}$ is a common condition associated with chronic elevation of right heart pressure as a result of chronic congestion with or without superimposed decrease in hepatic arterial perfusion [11]. Original characterization of histologic features of congestive hepatopathy was performed in necropsy or post-mortem specimens. The more widespread use of heart transplantation as a treatment for end stage of heart disease and the potential reversibility of congestive hepatopathy are associated with increased frequency of liver biopsies to assess the perioperative risk of these patients and/or to determine the need of synchronous liver transplant. However, large series of liver biopsies from patients with congestive heart failure are lacking.

Our study of 36 liver biopsies from patients with clinically and radiographically documented congestive heart failure revealed a spectrum of histology. The most common histologic finding in our series included sinusoidal dilation $(72 \%$ of cases), centribular fibrosis (69\% of cases), hepatocyte atrophy ( $58 \%$ of cases), and centrilobular hemorrhage ( $47 \%$ of cases). In addition, our study revealed the presence of mononuclear inflammation in the portal tracts and lobules in approximately $53 \%$ and $36 \%$ of cases, respectively. The overall finding is

Table 4. Correlation of Histologic Features and GS Staining Pattern in 24 Cases of Histologically Consensus Cardiac Hepatopathy

\begin{tabular}{lll} 
Histologic feature & Correlation coefficient & Significance (P value) \\
\hline Sinusoidal dilation (absent/present) & 0.209 & 0.317 \\
Centrilobular atrophy (absent/present) & 0.092 & 0.66 \\
Centrilobular hemorrhage (absent/present) & 0.192 & 0.356 \\
Centrilobular fibrosis (absent/present) & -0.126 & 0.546 \\
Fibrosis stage (congestive hepatic fibrosis score) & 0.107 & 0.571 \\
\hline
\end{tabular}


consistent with previous reports [1-4, 7]. In line with previous observations, only four of 36 (11\%) liver biopsies from our cohort of congestive heart failure showed cirrhosis $[2,6]$.

One previous study proposed a CHFS as a novel histologic assessment of clinical severity [7]. However, the CHFS has not been studied for its interobserver variability. Our study revealed that the overall agreement on the diagnosis of $\mathrm{CH}$ and CHFS was moderate $(\mathrm{K}=0.55,95 \% \mathrm{CI}: 0.32-0.73)$ and fair $(\mathrm{K}=0.35,95 \%$ CI: $0.24-0.49)$, respectively. However, if the staging is simplified into advanced (bridging or cirrhosis) and/or non-advanced fibrosis, the interobserver agreement is improved to moderate category $(\mathrm{K}=0.45,95 \%$ CI: 0.31 - 0.61). Among the common features of $\mathrm{CH}$, sinusoidal dilatation showed substantial interobserver agreement, followed by centrilobular hepatocyte atrophy and fibrosis (moderate agreement), and centrilobular hemorrhage (fair agreement).

One interesting finding is the low rate of steatosis in $\mathrm{CH}$ as $94 \%$ of $\mathrm{CH}$ had no steatosis or less than $5 \%$ steatosis in our study, lower than general population and young adults in the United States [12-14]. Whether this is related to the patient's overall nutrition status or due to impaired triglyceride synthesis in hepatocytes due to congestion with or without superimposed hepatic arterial perfusion remains unclear. Similarly, hepatocyte ballooning, Mallory hyalines, cholestasis, and neutrophilic inflammation are only rarely observed in $\mathrm{CH}$. The presence of these features should prompt searching for other etiologies such as alcohol, sepsis, biliary obstruction, and drug-induced liver injury.

As one small study previously suggested a potential use of GS in the diagnosis of $\mathrm{CH}$ [9], we performed immunohistochemistry of GS on a cohort of histologically confirmed cases of $\mathrm{CH}$. In contrast to the previous study, only $50 \%$ (12 out 24 ) of our cases had decreased perivenular GS expression. This may be due to stage difference and specimen type (100\% biopsy in the current study versus $75 \%$ (eight of 12 ) biopsy), use of different antibody, and cutoff value. Regardless, significant loss of perivenular GS expression may be a marker for $\mathrm{CH}$, but only with a sensitivity of $50 \%$. Interestingly, the loss of perivenular GS expression was not associated with the features we examined which include sinusoidal dilatation, centrilobular atrophy, centrilobular hemorrhage, and centrilobular fibrosis, and fibrosis (CHFS).

One of the main strengths of this study is that it is one of the largest cohorts of patients with $\mathrm{CH}$ from a single institute and all cases were confirmed clinically and radiographically. In addition, 24 cases of $\mathrm{CH}$ used for GS immunohistochemical study were agreed upon by at least three out of four liver pathologists who were GI and liver pathology fellowship trained and are actively practicing liver pathology.

There are limitations to our study. While these $\mathrm{CH}$ cases were clinically confirmed, the histology results including the CHFS were not correlated with detailed clinical features and demographics, lab test results, and the patients' outcome. The $\mathrm{CH}$ population in this cohort may be different from the cohort in previous studies or from other institutes as these cases were seen in our tertiary hospital before heart transplantation and our patients may potentially have more severe disease. In addition, this study only involves four liver pathologists and no general pathologists without liver pathology fellowship train- ing were included in this study.

In summary, we examined for the first time, the interobserver variability on several previously reported important histologic features of $\mathrm{CH}$ in a clinical practice outside of research setting. A CHFS could only be assigned with a fair level of agreement (kappa value of 0.35) in our study. Our data reaffirm the significant interobserver variability in liver biopsy interpretation in $\mathrm{CH}$ even among liver pathologists. Simplification of stage into advanced (bridging and cirrhosis) and nonadvanced fibrosis results in moderate interobserver agreement (kappa value of 0.45). Our current results suggest that implementing this scoring system can be challenging, especially for early stages of $\mathrm{CH}$.

\section{Disclosure}

None.

\section{Grant Support}

None.

\section{References}

1. Sherlock S. The liver in heart failure; relation of anatomical, functional, and circulatory changes. Br Heart J. 1951;13(3):273-293.

2. Lefkowitch JH, Mendez L. Morphologic features of hepatic injury in cardiac disease and shock. J Hepatol. 1986;2(3):313-327.

3. Arcidi JM Jr, Moore GW, Hutchins GM. Hepatic morphology in cardiac dysfunction: a clinicopathologic study of 1000 subjects at autopsy. Am J Pathol. 1981;104(2):159166.

4. Wanless IR, Liu JJ, Butany J. Role of thrombosis in the pathogenesis of congestive hepatic fibrosis (cardiac cirrhosis). Hepatology. 1995;21(5):1232-1237.

5. Dichtl W, Vogel W, Dunst KM, Grander W, Alber HF, Frick M, Antretter H, et al. Cardiac hepatopathy before and after heart transplantation. Transpl Int. 2005;18(6):697702.

6. Myers RP, Cerini R, Sayegh R, Moreau R, Degott C, Lebrec D, Lee SS. Cardiac hepatopathy: clinical, hemodynamic, and histologic characteristics and correlations. Hepatology. 2003;37(2):393-400.

7. Dai DF, Swanson PE, Krieger EV, Liou IW, Carithers RL, Yeh MM. Congestive hepatic fibrosis score: a novel histologic assessment of clinical severity. Mod Pathol. 2014;27(12):1552-1558.

8. Gieling RG, Ruijter JM, Maas AA, Van Den Bergh Weerman MA, Dingemans KP, ten Kate FJ, Lekanne dit Deprez $\mathrm{RH}$, et al. Hepatic response to right ventricular pressure overload. Gastroenterology. 2004;127(4):1210-1221.

9. Fleming KE, Wanless IR. Glutamine synthetase expression in activated hepatocyte progenitor cells and loss of 
hepatocellular expression in congestion and cirrhosis. Liver Int. 2013;33(4):525-534.

10. Landis JR, Koch GG. The measurement of observer agreement for categorical data. Biometrics. 1977;33(1):159-174.

11. Megalla S, Holtzman D, Aronow WS, Nazari R, Korenfeld S, Schwarcz A, Goldberg Y, et al. Predictors of cardiac hepatopathy in patients with right heart failure. Med Sci Monit. 2011;17(10):CR537-541.

12. Sayiner M, Koenig A, Henry L, Younossi ZM. Epidemiology of nonalcoholic fatty liver disease and nonalcohol- ic steatohepatitis in the United States and the rest of the world. Clin Liver Dis. 2016;20(2):205-214.

13. Kanwal F, Kramer JR, Duan Z, Yu X, White D, El-Serag HB. Trends in the burden of nonalcoholic fatty liver disease in a United States cohort of Veterans. Clin Gastroenterol Hepatol. 2016;14(2):301-308.e2.

14. Mrad RA, Merjaneh N, Mubarak G, Lopez R, Zein NN, Alkhouri N. The increasing burden of nonalcoholic fatty liver disease among young adults in the United States: A growing epidemic. Hepatology. 2016;64(4):1386-1387. 